\title{
Why Is the Diagnosis of Vestibular Disorders So Tricky?
}

Vertigo and dizziness represent a frequent complaint for which patients seek medical help. For several reasons, the diagnosis is often a difficult challenge for the clinician. First, the possible causes are multiple, including inner ear, central nervous system, cardiovascular, metabolic disorders, as well as psychological distress. Second, some typical features of a specific syndrome are sometimes absent for a long period of time. In addition, hearing loss, tinnitus and vertigo are unspecific subjective symptoms whose description differs considerably for each individual. Therefore, the clinical history, although an essential step for the diagnosis, is not always easy to obtain. Vertigo and imbalance represent such a frightening experience that some patients do not clearly make the difference between a true spell of vertigo and the fear it generates. In a few of them, the confusion between the multiple aspects of the illness, i.e. somatic, psychological, and socio-professional, is even such that psychological distress persists long after the vertigo has been definitely cured. Third, all the routinely used diagnostic tests have severe limitations. The caloric test does not represent a physiological stimulation and its response may be normal in severely affected patients or, on the contrary, abolished in symptom-free patients. Sinusoidal rotational tests evaluate the vestibulo-ocular reflex (VOR) in response to low-frequency stimulations in the horizontal plane only and do not always reveal the actual function of the vestibular system. Of course, systems allowing for a test of the vestibular responses to physiological high-frequency accelerations exist but they require powerful devices that are not widely available. So are systems which make it possible to evaluate the function of otolithic end organs. Recently, some systems measuring the VOR during active high-frequency head rotations in the horizontal and vertical planes have been marketed, but they have been shown to have poor test-retest reliability.
To establish a diagnosis, the clinician can choose between two options. He or she may perform systematically a standardized otoneurological workup with audiological and vestibular testings, and a CT scan or MRI in all the patients complaining of vertigo and/or disequilibrium. This option can, however, induce unecessary examinations or produce results that are inconsistent because of the limitations of the diagnostic tests rather than an actual anomaly of the vestibular system. On the other hand, the clinician may choose to limit the investigations to the minimal useful tests. The risk with this option is that information indispensable to the diagnosis may be missed. As an example, patients with an acoustic neuroma may have normal pure tone audiogram, brainstem auditory evoked potentials, and caloric response.

In this issue, experts were invited to debate on the minimal useful tests in patients complaining of vertigo or dizziness. Most underline that the clinical history, a careful physical exam and some simple tests are the most essential elements of the diagnosis. They also show that multiple sophisticated tests are under development or already exist but still cannot be routinely used in clinical practice for many different reasons. However, some questions have not been discussed, i.e. in which cases a CT and/or an MRI is necessary, the need for serological examinations to better understand the etiology of the underlying cause, and the value of the many questionnaires developed during the last decade to evaluate the quality of life and the functional ability of patients suffering from otoneurological disorders. These questions are still open and we expect that they as well as some of the opinions expressed in this issue will draw additional comments from readers, and we will be pleased to publish these in the next issue of Oto-Rhino-Laryngologia Nova.

Jean-Philippe Guyot, Geneva

\begin{tabular}{ll}
\hline KARGER & ( ) 2002 S. Karger AG, Basel \\
Fax +4161306 1234-8221/01/0112-0057\$17.50/0 \\
$\begin{array}{l}\text { E-Mail karger@karger.ch } \\
\text { www.karger.com }\end{array}$ & $\begin{array}{l}\text { Accessible online at: } \\
\text { www.karger.com/journals/orn }\end{array}$
\end{tabular}

